\title{
A 1.5-V, 2.4GHz CMOS Low-Noise Amplifier
}

\author{
Jyh-Neng Yang, Chen-Yi Lee, Terng-Yin Hsu, Terng-Ren Hsu, Chung-Cheng Wang \\ Department of Electronics Engineering And Institute of Electronics \\ National Chiao Tung University \\ Hsin-Hsing Rd., Hsin-Fong, Hsin-Chu 304, Taiwan R.O.C.
}

Tel: 886-3-5593769 Fax: 886-3-5573177 e-mail: yjn@mhit.edu.tw

\begin{abstract}
A 2.4GHz low noise amplifier has been designed in a standard CMOS 0.35 um process. The transistor model is Bsim3 for 0.35um process. The amplifier provides a forward gain of $33 \mathrm{~dB}$ with a noise figure only $0.92 \mathrm{~dB}$ while drawing $17 \mathrm{mw}$ from a $1.5 \mathrm{~V}$ supply. Design simulation results are presented in this paper.
\end{abstract}

\section{INTRODUCTION}

As we know, the first stage of receiver is commonly a low-noise amplifier (LNA), whose main function is to provide enough gain to overcome the noise of subsequent stages (such as mixers), but not so much to cause the mixer overload. Secondly, an LNA should add as little noise as possible to minimize the impact on the overall noise performance. Thirdly, an LNA should accommodate large signals without distortion, i.e., provide good linearity. Frequently, an LNA also must provide specific impedance, such as 50 Ohms, to the input source and output load, which are particularly important. In addition, an LNA should provide low power consumption especially in portable systems.

The design of an LNA is full trade-offs between optimum gain, lowest noise figure, optimum input and output matching, high linearity and lower power consumption. While the literature is full of examples of LNA work in GaAs and bipolar technologies, there are few examples of CMOS design. However, CMOS provides the possibility of the integration of complete communication systems. Only recently have more and more people been involved in CMOS LNA designs, and have subsequently demonstrated the viability of CMOS LNA from $900 \mathrm{MHz}$ to 2.0GHz. [1][2][3].

\section{CIRCUIT DESIGN}

Following the circuit design given in [1][2], and with details presented in another conference [4], the complete circuit is shown in Figure 1. It was found that two-stage amplifier was required to achieve the desired gain, and provided good isolation between the input and output. The first stage is a cascode amplifier form of M1 and M2. Ls and $\mathrm{Lg}$ are for input matching. $\mathrm{Ld}$ and $\mathrm{Cd}$ form a tank circuit to tune the LNA to $2.4 \mathrm{GHz}$. M3 is output stage with matching network of Lo and $\mathrm{Co}$. MO, Rd and $\mathrm{Rg}$ form a bias circuit. M0 essentially is a current mirror with $M 1$, while $\mathrm{Rg}$ prevents the bias circuit from shunt the RF input signal. $\mathrm{Ci}$ is used as a DC blocking capacitor. P1 and P2 are $50 \mathrm{Ohms}$ source and load ports. On chip components include all transistors, inductors, capacitors and resistors.

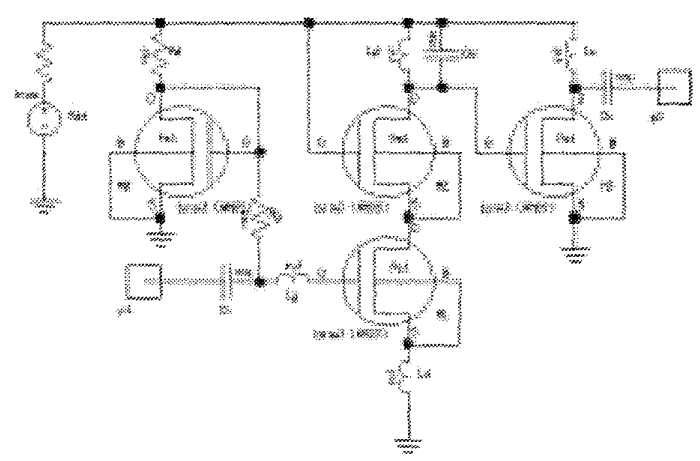

Figure 1. Complete Circuit

\section{SIMULATION RESULTS}

The proposed circuit was simulated using Serenade 8.5 . The transistor model is Bsim 3v3 for TSMC 0.35 um process. Figure 2 shows the forward gain of LNA. The amplifier provides a forward gain of $33 \mathrm{~dB}$ at $2.4 \mathrm{GHz}$. Noise Figure (NF) is obtained and shown in Figure 3 . The simulated LNA achieves a noise figure of $0.9 \mathrm{~dB}$ at $2.4 \mathrm{GHz}$. The $\mathrm{S} 11$ and S22 parameters of LNA are illustrated in Figure 4 . The simulated $\mathrm{S} 11$ and $\mathrm{S} 22$ are $-23 \mathrm{~dB},-9 \mathrm{~dB}$ respectively. The linearity of the LNA, which is normally evaluated by the output referred third-order intercept point (OIP3), is plotted in Figure 5. The simulated OIP3 is around $25 \mathrm{dBm}$. Figure 6 show the response of input power versus output power, which indicates the $1 \mathrm{~dB}$ compression point. The simulated $1 \mathrm{~dB}$ compression point is $-26 \mathrm{dBm}$. The complete layout of 
the LNA circuit is shown in Figure 7, was fabricated by using TSMC 0.35 um CMOS technology with a die size of $850 \mathrm{um} \times 600 \mathrm{um}$. The LNA drains $10.8 \mathrm{~mA}$ from a $1.5 \mathrm{~V}$ supply, dissipates $16.2 \mathrm{~mW}$

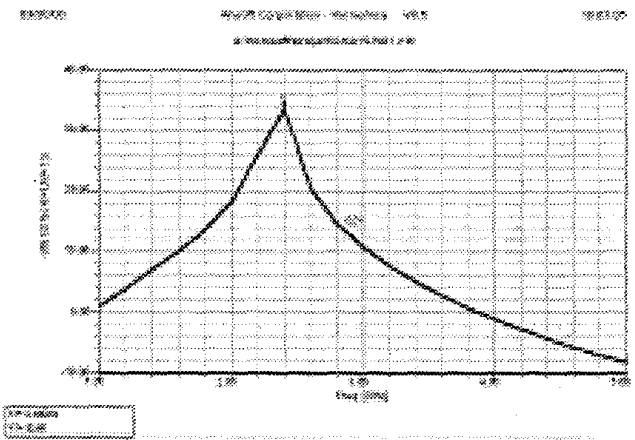

Figure 2. Forward Gain S21 of LNA

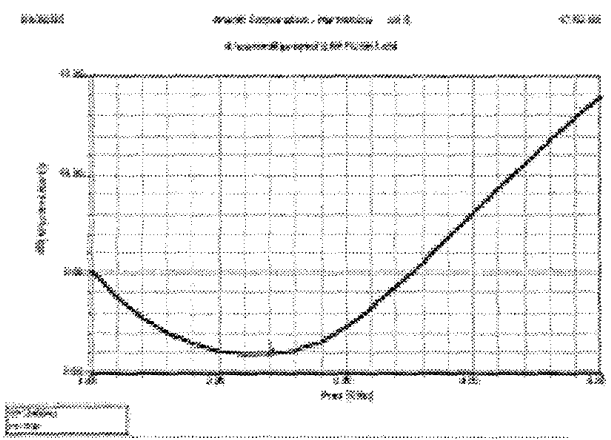

Figure 3. Noise Figure (NF)

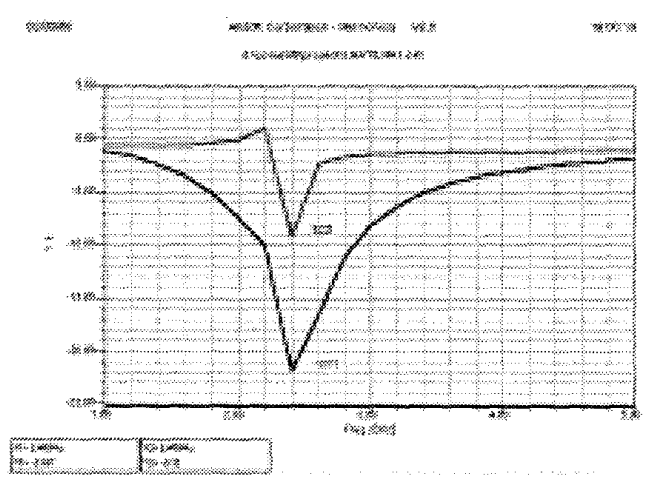

Figure 4. S-parameter (S11, S22)

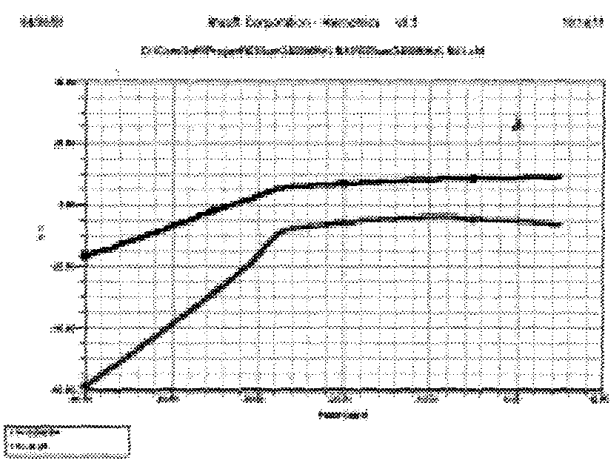

Figure5. The response of OIP3

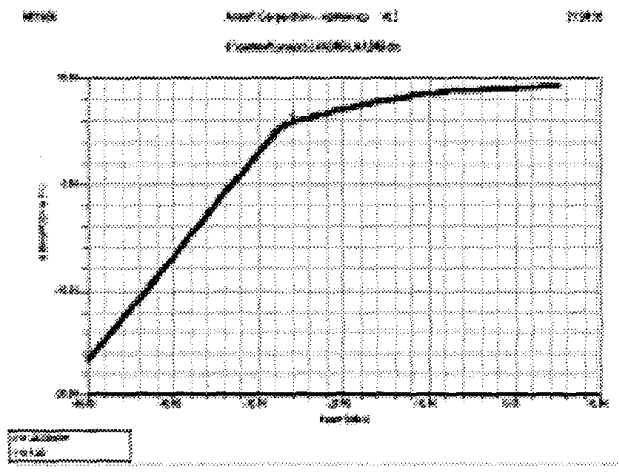

Figure 6. The response of input power versus output power

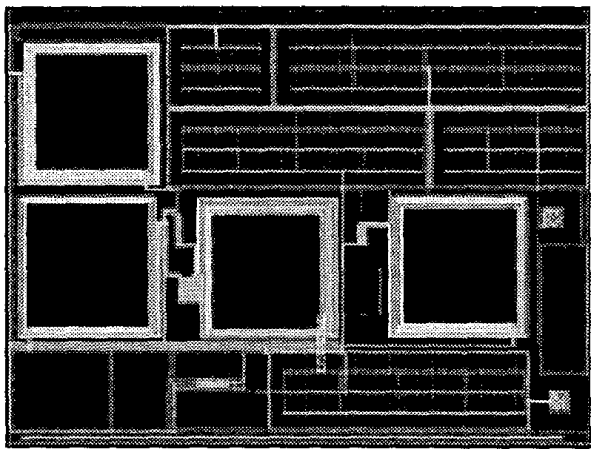

Figure 7 Layout of proposed LNA 


\section{CONCLUSION}

In this paper, we have demonstrated a low noise amplifier in a standard CMOS 0.35 um process, which can be the first amplifier in the receiver of a portable system. Based on the simulation results, we believe that CMOS is a suitable technology for wireless receiver design and will provide the integration of a complete communication system on a single chip in the future.

\section{REFERENCE}

[1] Lee, T. H., "The Design of CMOS Radio-Frequency Integrated Circuit", Cambridge University Press, 1998.

[2] Shaeffer, D. K. and Lee, T. H., "A 1.5V 1.5 GHz CMOS Low Noise Amplifier", IEEE J. of Solid-State Circuit, Vol. 32, N0. 5, May 1997, page 745-759.

[3] Yang, S., Mason, R. and Plett, C., "6.5mW CMOS Low Noise Amplifier at $1.9 \mathrm{GHz}$," to be presented in IEEE International Symposium on Circuit and System, Orlando, Florida, 1999.

[4] Karanicolaa, A. N., "A 2.7V 900MHz CMOS LNA and mixer," IEEE ISSCC Proceeding, 1996, page 50-51. 